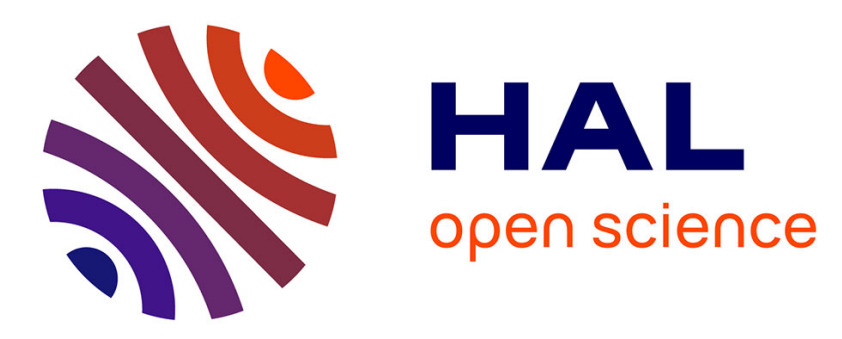

\title{
A new paradigm for the optimum design of variable angle tow laminates \\ Marco Montemurro, Anita Catapano
}

\section{To cite this version:}

Marco Montemurro, Anita Catapano. A new paradigm for the optimum design of variable angle tow laminates. Variational analysis and aerospace engineering: mathematical challenges for the aerospace of the future, 116, Springer, 2016, Springer Optimization and Its Applications, 978-3-319-45679-9. hal-01354284

\section{HAL Id: hal-01354284 \\ https://hal.science/hal-01354284}

Submitted on 18 Aug 2016

HAL is a multi-disciplinary open access archive for the deposit and dissemination of scientific research documents, whether they are published or not. The documents may come from teaching and research institutions in France or abroad, or from public or private research centers.
L'archive ouverte pluridisciplinaire HAL, est destinée au dépôt et à la diffusion de documents scientifiques de niveau recherche, publiés ou non, émanant des établissements d'enseignement et de recherche français ou étrangers, des laboratoires publics ou privés. 


\section{A new paradigm for the optimum design of variable angle tow laminates \\ Marco Montemurro* \\ Arts et Métiers ParisTech, I2M CNRS UMR 5295, F-33400 Talence, France. \\ Anita Catapano \\ Bordeaux INP, Université de Bordeaux, I2M CNRS UMR 5295, F-33400 Talence, France}

Author for Correspondence:

Marco Montemurro, PhD,

Arts et Métiers ParisTech,

I2M CNRS UMR 5295,

F-33400 Talence, France,

tel: +33556845422 ,

fax: +33540006964 ,

e.mail: marco.montemurro@ensam.eu,

marco.montemurro@u-bordeaux1.fr

${ }^{*}$ Corresponding author. 


\begin{abstract}
In this work the authors propose a new paradigm for the optimum design of variable angle tow (VAT) composites. They propose a generalisation of a multi-scale two-level (MS2L) optimisation strategy already employed to solve optimisation problems of anisotropic structures characterised by a constant stiffness distribution. In the framework of the MS2L methodology, the design problem is split into two sub-problems. At the first step of the strategy the goal is to determine the optimum distribution of the laminate stiffness properties over the structure, while the second step aims at retrieving the optimum fibres-path in each layer meeting all the requirements provided by the problem at hand. The MS2L strategy relies on: a) the polar formalism for describing the behaviour of the VAT laminate, b) the iso-geometric surfaces for describing the spatial variation of the stiffness properties and c) an hybrid optimisation tool (genetic and gradient-based algorithms) to perform the solution search. The effectiveness of the MS2L strategy is proven through a numerical example on the maximisation of the first buckling factor of a VAT plate subject to both mechanical and manufacturability constraints.
\end{abstract}

\title{
Keywords:
}

Composite materials; Variable Stiffness; Anisotropy; Variable Angle Tow Laminates; Buckling; Computational modelling; Optimisation.

\section{Introduction}

Anisotropic materials, such as fibre reinforced composite materials, are extensively used in many industrial fields thanks to their mechanical performances: high stiffness-to-weight and strength-to-weight ratios that lead to a substantial weight saving when compared to metallic alloys. In addition, the recent development of new manufacturing techniques of composite structures, e.g. automated fibre-placement (AFP) machines, allows for going beyond the classical design rules, thus leading the designer to find innovative and more efficient solutions than the classical straight fibres configurations. The use of the AFP technology brought to the emergence of a new class of composite materials: the variable angle tow (VAT) composites, [10, 12]. A modern AFP machine allows the fibre (i.e. the tow) to be placed along a curvilinear path within the constitutive lamina thus implying a point-wise variation of the material properties (stiffness, strength, etc.). Of course, this technology enables the designer to take advantage of the directional properties of composites in the most effective way. The interest of using variable stiffness (VS) laminates is considerably increased during the last years: in the meantime some works on the $a$ posteriori characterisation of the elastic response of such materials have gained a lot of attention from the scientific community of composites materials. For example [7] deals with the problem of predicting the impact and compression after impact behaviour of VAT laminates while [33] analyses the pre-buckling and buckling mechanisms in VAT laminated plates through a proper evaluation of the non-uniform stress variation within the structure 
due to the variable stiffness distribution. Although the utilisation of VAT laminates considerably increases the complexity of the design process (mainly due to the large number of design variables involved within the problem), on the other hand it leads the designer to conceive non-conventional solutions characterised either by a considerable weight saving or enhanced mechanical properties when compared to classical solutions, [26, 27, 28, 29]. One of the first works that tried to explore the advantages that can be achieved in terms of mechanical performances (stiffness, buckling behaviour, etc.) by using a VS plate in which each ply is characterised by a curvilinear path of the tow (i.e. a VAT configuration) instead of the conventional straight-line fibre format is presented in [12]. The authors make use of a sensitivity analysis and a gradient-based search technique to determine the optimal fibre orientation in a given number of regions of the plate. This work proved that a considerable increment of the buckling load of the structure can be obtained when employing a VAT solution for the layered plate.

The complexity of the design process of a VAT laminated structure is mainly due to two intrinsic properties of VAT composites, i.e. the heterogeneity and the anisotropy that intervene at different scales of the problem and that vary point-wise over the structure. Moreover, a further difficulty is due to the fact that the problem of (optimally) designing a VAT laminate is intrinsically a multi-scale design problem. Indeed, in order to formulate the problem of designing a VAT composite in the most general way, the designer should take into account, within the same design process, the full set of design variables (geometrical and material) governing the behaviour of the structure at each characteristic scale (micromeso-macro). Up to now no general rules and methods exist for the optimum design of VAT laminates. Only few works on this topic can be found in literature, and all of them always make use of some simplifying hypotheses and rules to get a solution. An exhaustive review focusing on constant and variable stiffness design of composite laminates is presented in $[8,9]$. In [1] the first natural frequency of VS composite panels is maximised by considering on the one hand the lamination parameters and the classical laminate theory (CLT) for the description of the local stiffness properties of the structure and, on the other hand, a generalised reciprocal approximation algorithm for the resolution of the optimisation problem. This approach is limited to the determination of the stiffness properties of an equivalent homogenised plate, since the lay-up design phase is not at all considered. In [30] the least-weight design problem of VAT laminates submitted to constraints including the strength and the radius of curvature is considered. The design variables are the layers thickness and fibres angles which are represented by bi-cubic Bezier surfaces and cubic Bezier curves, respectively. A sequential quadratic programming method is used to solve the optimisation problem. A two-level strategy was employed in [34] to design a VAT laminated plate by determining firstly a discretised optimal distribution of lamination parameters and secondly an optimum lay-up corresponding to the local distribution of 
lamination parameters. [34] represents the first attempt of applying a multi-scale numerical strategy (i.e. a two-level design procedure) which aims at determining, at the first level, the optimum local (i.e. point-wise) distribution of the stiffness properties of the structure (in terms of the lamination parameters of the laminate), while at the second level the optimum path (in each constitutive layer) corresponding to the optimal distribution of the lamination parameters resulting from the first step. However, the major drawback of this work actually was in the determination of the curvilinear fibres-path of each layer: the resulting path was discontinuous because the authors had not foreseen a numerical strategy able to simultaneously meet on the one hand the continuity of the fibres path (between adjacent elements) and on the other hand the optimum distribution of lamination parameters provided by the first step of the procedure. A further work on the same topic can be found in [35] where the problem of designing variable-stiffness composite panels for maximum buckling load is addressed by making use of the generalised reciprocal approximation approach introduced by Abdalla [1]. In [35] the the two-level approach was abandoned and the authors stated the problem by directly considering the fibre orientation angles in each ply and for each element of the plate as design variables. However, as in [1], this approach always leads to a discontinuous fibre path and, unlike the strategy proposed in [1], it leads also to the emergence of a new issue: the resulting optimisation problem was highly non-convex since it was formulated directly in the space of the layer orientations (which vary locally over the plate). Accordingly, in [35] the authors conclude that such an issue can be potentially remedied by formulating in a proper way the design problem of VAT laminates in the framework of the two-level strategy and by trying to take into account the issue of the continuity of the fibres path directly in the first level of the strategy where the design variables are the laminate mechanical properties (i.e. the lamination parameters in the theoretical framework of $[34,35])$.

Another issue often addressed by researches on VAT laminates concerns the tow placement technology which could introduce several differences (i.e. imperfections) between the numerical model of the VAT composite and the real structure tailored with the AFP process, if the design methodology does not take into account the manufacturability requirements. To this purpose in [3] an issue linked to the AFP technology is addressed: the overlap of tow-placed courses that increases the ply thickness (the build-up phenomenon) thus affecting the structural response and surface quality of the laminate. The work of Blom et al. [3] presents a method for designing composite plies with varying fibre angles. The fibre angle distribution per ply is given while, using a streamline analogy, the optimal distributions of fibre courses is determined for minimising the maximum ply thickness or maximising the surface smoothness. An improved research on this topic has been developed in [31] where an algorithm is presented to optimise the fibre path in order to ensure manufacturability. A further work focusing on the development and/or improvement of 
manufacturing techniques for tailoring VAT laminates in order to minimise the imperfections induced by the fabrication process is presented in [14]. The continuous tow shearing (CTS) technique, utilising the ability to shear dry tows, is proposed as an alternative technique to the well-known AFP process. Later, the work presented in [14] has been improved through the introduction of a computer-aided modelling tool [15] which can create accurate finite element models reflecting the fibre trajectories and thickness variations of VAT composites manufactured using the CTS technique.

As a summary of this non-exhaustive review on VAT composites it can be stated that the main limitations and drawbacks characterising the vast majority of the studies on these materials are:

- discontinuous distribution of the material parameters (e.g. lamination parameters) describing the elastic response of the laminate over the structure;

- discontinuous distribution of the local fibres orientation angle within each ply;

- the use of linear/quadratic functions for representing the fibre path (which significantly reduces the design domain);

- the lack of a proper and efficient multi-scale approach for dealing with the (optimal) design problem of VAT laminates;

- the absence of practical rules for taking into account the manufacturability/ technological constraints since the early stages of the design process;

- the applications which are limited only to "academic" cases and not extended to real-world engineering problems.

To overcome the previous restrictions the present work focuses mainly on the generalisation and extension of the multi-scale bi-level (MS2L) procedure for the optimum design of composite structures (initially introduced in $[19,20]$ ) to the case of VAT composites. The idea of a bi-level (or multi-level) procedure for designing composite structures is not entirely new and has already been used in the past [11]. Up to now this strategy has been employed only by few authors for the optimisation of composite structures but in each study the link between the levels of the procedure and the scales of the problem was never rigorously stated.

The authors and their co-workers already made use of the MS2L procedure for the design and optimisation of several classes of hybrid anisotropic structures in the past $[4,5$, $6,17,18,19,20,25]$. The MS2L design strategy employed in the previous works is a very general methodology for designing composites structures: it is characterised on the one hand by the refusal of the simplifying hypotheses and classical rules usually employed in the framework of the design process of laminates, and on the other hand by a proper and 
complete mathematical formalisation of the optimum design problem at each characteristic scale (micro-meso-macro). The MS2L strategy relies on the use of the polar formalism (initially introduced by Verchery [39], and later extended to the case of higher-order theories $[22,23,24])$ for the description of the anisotropic behaviour of the composite. The real advantage in using the Verchery's polar method within the design process of composite structures is in the fact that the elastic response of the structure at the macro-scale is described in terms of tensor invariants, the so-called polar parameters having a precise physical meaning (which is linked to the elastic symmetries of the material) [37]. On the other hand the MS2L strategy relies on the use of a particular genetic algorithm (GA) able to deal with a special class of huge-size optimisation problems (from hundreds to thousands of design variables) defined over a domain of variable dimension, i.e. optimisation problems involving a "variable number" of design variables [17].

As far as concerns the problem of designing VAT composites, the aim of this paper is twofold. On the one hand a new paradigm for designing VAT laminates is introduced, while on the other hand the MS2L optimisation strategy has been generalised in order to deal with the design problem of VAT composites. Several modifications have been introduced in the theoretical and numerical framework of the MS2L design procedure at both the first and second levels. At the first level (laminate macroscopic scale) of the procedure, where the VAT laminate is modelled as an equivalent homogeneous anisotropic plate whose mechanical behaviour is described in terms of polar parameters (which vary locally over the structure) the major modifications are: 1) the utilisation of higher-order theories (First-order Shear Deformation Theory (FSDT) framework [22, 23]) for taking into account the influence of the transverse shear stiffness on the overall mechanical response of VAT composites; 2) the utilisation of B-spline surfaces for obtaining a continuous point-wise variation of the laminate polar parameters. Regarding the second-level problem (laminate mesoscopic scale, i.e. the ply level) the main modifications are: 1) the utilisation of B-spline surfaces for obtaining a continuous point-wise variation of the fibre orientation angle within each ply; 2) a proper mathematical formalisation of the manufacturability constraints linked to the AFP process in the framework of the B-spline representation. All of these modifications imply several advantages for the resolution of the related optimisation problems (both at first and second level of the strategy) that will be detailed in Sections 3 and 4.

The paper is organised as follows: the design problem and the MS2L strategy are discussed in Section 2. The mathematical formulation of the first-level problem is detailed in Section 3, while the mathematical statement of the second-level problem (the lay-up design) is presented in Section 4. A concise description of the Finite Element (FE) model of the VAT layered plate is given in Section 5, while the numerical results of the optimisation procedure are shown in Section 6. Finally, Section 7 ends the paper with some concluding 
remarks.

\section{A new design paradigm for VAT laminates}

\subsection{Description of the problem}

The optimisation strategy presented in this study is applied to a VAT laminated plate composed of a fixed number of plies, hence the total thickness of the plate is fixed a priori. The fibre tow is made of carbon-epoxy pre-preg strips whose elastic properties are listed in Table 1.

\begin{tabular}{|c|c|c|c|c|c|c|}
\hline \multicolumn{2}{|c|}{ Technical constants } & \multicolumn{2}{|c|}{ Polar parameters of $[\mathrm{Q}]^{a}$} & \multicolumn{2}{|c|}{ Polar parameters of $[\hat{Q}]$} & $b$ \\
\hline$E_{1}[\mathrm{MPa}]$ & 161000.0 & $T_{0}[\mathrm{MPa}]$ & 23793.3868 & $T$ [MPa] & 5095.4545 & \\
\hline$E_{2}[\mathrm{MPa}]$ & 9000.0 & $T_{1}[\mathrm{MPa}]$ & 21917.8249 & $R[\mathrm{MPa}]$ & 1004.5454 & \\
\hline$G_{12}[\mathrm{MPa}]$ & 6100.0 & $R_{0}[\mathrm{MPa}]$ & 17693.3868 & $\Phi$ [deg] & 90.0 & \\
\hline$\nu_{12}$ & 0.26 & $R_{1}[\mathrm{MPa}]$ & 19072.0711 & & & \\
\hline \multirow[t]{2}{*}{$\nu_{23}$} & 0.10 & $\Phi_{0}[\mathrm{deg}]$ & 0.0 & & & \\
\hline & & $\Phi_{1}[\mathrm{deg}]$ & 0.0 & & & \\
\hline $\begin{array}{l}\text { Density and } \\
\rho\left[\mathrm{Kg} / \mathrm{mm}^{3}\right] \\
h_{p l y}[\mathrm{~mm}]\end{array}$ & $\begin{array}{l}\text { hickness } \\
1.58 \times 10^{-6} \\
0.125\end{array}$ & & & & & \\
\hline
\end{tabular}

${ }^{a}$ In-plane reduced stiffness matrix of the pre-preg strip.

${ }^{b}$ Out-of-plane shear stiffness matrix of the pre-preg strip.

Table 1: Material properties of the carbon-epoxy pre-preg strip, see [22, 23].

Concerning the mechanical behaviour of the VAT plate, further details have to be added in order to clearly define the theoretical framework of this work:

- the geometry of the laminated structure and the applied Boundary Conditions (BCs) are known and fixed;

- the VAT plate is composed of identical plies (i.e. same material and thickness);

- the material behaviour is linear elastic;

- the VAT plate is quasi-homogeneous and fully orthotropic $[4,5,25]$ point-wise, i.e. these properties apply locally in each point of the structure;

- at the macro-scale (i.e. the scale of the structure) the elastic response of the VAT plate is described in the theoretical framework of the FSDT and the stiffness matrices of the plate (whose components vary point-wise over the structure) are expressed in terms of the laminate polar parameters [22, 23] which constitute also the design variables of the VAT plate at the macroscopic scale. 
As far as concerns the mesoscopic scale of the VAT laminate (i.e. that of the constitutive ply) no simplifying hypotheses are made on the rest of the design parameters of the laminated plate, i.e. the design variables of the stack, namely the layer position and orientation angle (which varies point-wise for each layer). Only avoiding the utilisation of a priori assumptions that extremely shrink the solution space (e.g. the utilisation of symmetric balanced stacks to attain membrane/bending uncoupling and membrane orthotropy, respectively) one can hope to obtain the true global optimum for a given problem: this is a key-point in the proposed approach.

\subsection{Description of the multi-scale two-level optimisation strategy}

The main goal of the design strategy is the maximisation of the first buckling load of a VAT plate subject to

- feasibility constraints on the material parameters (i.e. the laminate polar parameters) governing the behaviour of the structure at the macroscopic scale;

- manufacturability constraints on the local radius of the tow (i.e. the local steering) due to the considered AFP technology.

The optimisation procedure is articulated into the following two distinct (but linked) optimisation problems.

1. First-level problem. The aim of this phase is the determination of the optimum distribution of the material properties of the VAT structure in order to minimise the considered objective function and to meet, simultaneously, the full set of optimisation constraints provided by the problem at hand. At this level the VAT plate is modelled as an equivalent homogeneous anisotropic continuum whose behaviour at the macro-scale is described in terms of laminate polar parameters, in the theoretical background of the FSDT [22, 23], which vary point-wise over the structure. Indeed the distributions of the laminate polar parameters over the laminated plate constitute the design variables of the first-level problem.

2. Second-level problem. The purpose of this design phase is the determination of the optimum lay-up of the laminate composing the structure (the laminate mesoscale) meeting the optimum combination of the polar parameters provided by the first level of the strategy. At this stage, the design variables are the layer orientation angles which vary point-wise in each ply (namely the fibre path) and, if needed, at this stage the designer can add some additional requirements, e.g. constraints on the elastic behaviour of the laminate, manufacturability constraints, strength and damage criteria, etc. 
To the best of the authors knowledge only few research activities have been carried out on the application of the bi-level optimisation procedure to the design problem of VAT laminates $[6,30,36]$. Although these works focus only on "academic" cases and benchmarks, they prove that, for a given geometry of the considered structure, the utilisation of a VAT solution allows for obtaining superior mechanical characteristics when compared to a classical multilayer solution composed of unidirectional laminae. This result is due to the elastic behaviour of VAT laminates which fit point-wise the equivalent material properties to the stress and strain fields engendered within the structure. Despite some relevant advances illustrated in [6], the bi-level approach presented in that work for dealing with the problem of the optimum design of VAT composites suffer of the following drawbacks:

- the optimum solution resulting from the first step of the procedure often consists in a discontinuous distribution of the laminate polar parameters which results in a discontinuous fibres path (for each constitutive layer) for the second-level problem;

- the lack of practical rules and of a very general mathematical formulation for determining a proper fibres path;

- the manufacturability constraints linked to the AFP process are not taken into account within the design process (i.e. within the problem formulation in the context of the bi-level optimisation procedure).

Accordingly, the optimum solutions illustrated in [6] cannot be manufactured. In order to overcome the difficulties listed above, some major modifications have been introduced within the mathematical formulation of the design/optimisation problem of VAT composites (for each level of the MS2L strategy), especially for taking into account within the design process the manufacturability constraints related to the AFP process. These modifications are detailed for each level of the numerical optimisation strategy in Section 3 and 4 , respectively.

\section{Mathematical formulation of the first-level problem}

In order to apply the MS2L numerical optimisation strategy presented in $[5,25]$ to the case of VAT composites some major modifications have been introduced. Regarding the first-level problem these modifications focus on:

- the utilisation of higher-order theories (in this case the FSDT framework) for taking into account the influence of the transverse shear stiffness on the overall mechanical response of the VAT laminate;

- the utilisation of B-spline surfaces for expressing the variation of the laminate polar parameters over the structure. 
The first point represents a very important step forward in the MS2L strategy when applied to every kind of composite structure (classical or VAT) as it allows to properly design thin as well as moderately thick plates.

The second modification leads to important consequences, too. Such consequences constitute just as many advantages for the resolution of the related optimisation problem. Firstly, the utilisation of iso-geometric surfaces leads to a considerable reduction in the number of material design variables (at the macro-scale), i.e. the polar parameters defined in each point of the control net of the B-spline surface. Secondly, thanks to the strong convex hull property of the B-spline blending functions the optimisation constraints of the problem, related to the specifications of the considered application, can be imposed only on the control points of the net: if they are satisfied on such points they are automatically met over the whole domain.

As previously stated the goal of the first level of the strategy is the maximisation of the buckling load of the VAT laminate by simultaneously satisfying the feasibility constraints on the distribution of the laminate polar parameters over the plate. All of these aspects are detailed in the following subsection.

\subsection{Mechanical design variables}

In the framework of the FSDT theory [13] the constitutive law of the laminated plate (expressed within the global frame of the laminate $R=\{0 ; x, y, z\}$ ) can be stated as:

$$
\begin{gathered}
\left\{\begin{array}{l}
\{\mathrm{N}\} \\
\{\mathrm{M}\}
\end{array}\right\}=\left[\begin{array}{ll}
{[\mathrm{A}]} & {[\mathrm{B}]} \\
{[\mathrm{B}]} & {[\mathrm{D}]}
\end{array}\right]\left\{\begin{array}{c}
\left\{\varepsilon_{0}\right\} \\
\left\{\chi_{0}\right\}
\end{array}\right\}, \\
\{\mathrm{F}\}=[\mathrm{H}]\left\{\gamma_{0}\right\},
\end{gathered}
$$

where $[\mathrm{A}],[\mathrm{B}]$ and $[\mathrm{D}]$ are the membrane, membrane/bending coupling and bending stiffness matrices of the laminate, while $[\mathrm{H}]$ is the out-of-plane shear stiffness matrix. $\{\mathrm{N}\}$, $\{\mathrm{M}\}$ and $\{\mathrm{F}\}$ are the vectors of membrane forces, bending moments and shear forces per unit length, respectively, whilst $\left\{\varepsilon_{0}\right\},\left\{\chi_{0}\right\}$ and $\left\{\gamma_{0}\right\}$ are the vectors of in-plane strains, curvatures and out-of-plane shear strains of the laminate middle plane, respectively, [13].

In order to analyse the elastic response of the multilayer plate the best practice consists 
in introducing the laminate homogenised stiffness matrices defined as:

$$
\begin{aligned}
& {\left[\mathrm{A}^{*}\right]=\frac{1}{h}[\mathrm{~A}]} \\
& {\left[\mathrm{B}^{*}\right]=\frac{2}{h^{2}}[\mathrm{~B}]} \\
& {\left[\mathrm{D}^{*}\right]=\frac{12}{h^{3}}[\mathrm{D}]} \\
& {\left[\mathrm{H}^{*}\right]= \begin{cases}\frac{1}{h}[\mathrm{H}] & \text { (basic) } \\
\frac{12}{5 h}[\mathrm{H}] & \text { (modified) }\end{cases} }
\end{aligned}
$$

where $h$ is the total thickness of the laminated plate.

In the framework of the polar formalism it is possible to express the Cartesian components of these matrices in terms of their material invariants. To the best of the authors knowledge, in $[22,23]$ an invariant representation of the laminate stiffness matrices in the framework of the FSDT has been given for the first time.

These works make use of the polar formalism [39] that gives a representation of any planar elasticity-like tensor by means of a complete set of independent invariants, i.e. the polar parameters. It can be proven that, also in the FSDT theoretical framework, in the case of a fully orthotropic, quasi-homogeneous laminate the overall number of independent mechanical design variables describing the elastic response of the laminate reduces to only three $[22,23]$ : the anisotropic polar parameters $R_{0 K}^{A^{*}}$ and $R_{1}^{A^{*}}$ and the polar angle $\Phi_{1}^{A^{*}}$ (this last representing the orientation of the main orthotropy axis) of the homogenised membrane stiffness matrix $\left[\mathrm{A}^{*}\right]$. In fact, as explained in $[22,23]$, once the material of the constitutive ply is fixed, the number of polar parameters to be designed remains unchanged when passing from the theoretical framework of the CLT to that of the FSDT; this result is quite surprising and represents a further advantage coming from the utilisation of the polar method. For more details on the polar formalism and its application in the context of the FSDT the reader is addressed to [22, 23, 37].

For a VAT composite the three independent polar parameters (which completely describe the mechanical behaviour of the VAT laminate at the macroscopic scale) must vary point-wise over the structure. As stated beforehand, such a variation is expressed by means of B-spline surfaces. In particular, in the mathematical framework of the B-spline surfaces the variation of the laminate polar parameters can be expressed as:

$$
\begin{aligned}
R_{0 K}^{A^{*}}(\xi, \gamma) & =\sum_{i=0}^{n_{p}} \sum_{j=0}^{m_{p}} N_{i, p}(\xi) N_{j, q}(\gamma) R_{0 K}^{A^{*}(i, j)}, \\
R_{1}^{A^{*}}(\xi, \gamma) & =\sum_{i=0}^{n_{p}} \sum_{j=0}^{m_{p}} N_{i, p}(\xi) N_{j, q}(\gamma) R_{1}^{A^{*}(i, j)} \\
\Phi_{1}^{A^{*}}(\xi, \gamma) & =\sum_{i=0}^{n_{p}} \sum_{j=0}^{m_{p}} N_{i, p}(\xi) N_{j, q}(\gamma) \Phi_{1}^{A^{*}(i, j)}
\end{aligned}
$$


Eq. (4) fully describes a B-spline surface (in the space of the laminate polar parameters) of degrees $p$ and $q$ along the parametric coordinates $\xi$ and $\gamma$, respectively, as depicted in Fig. 1.

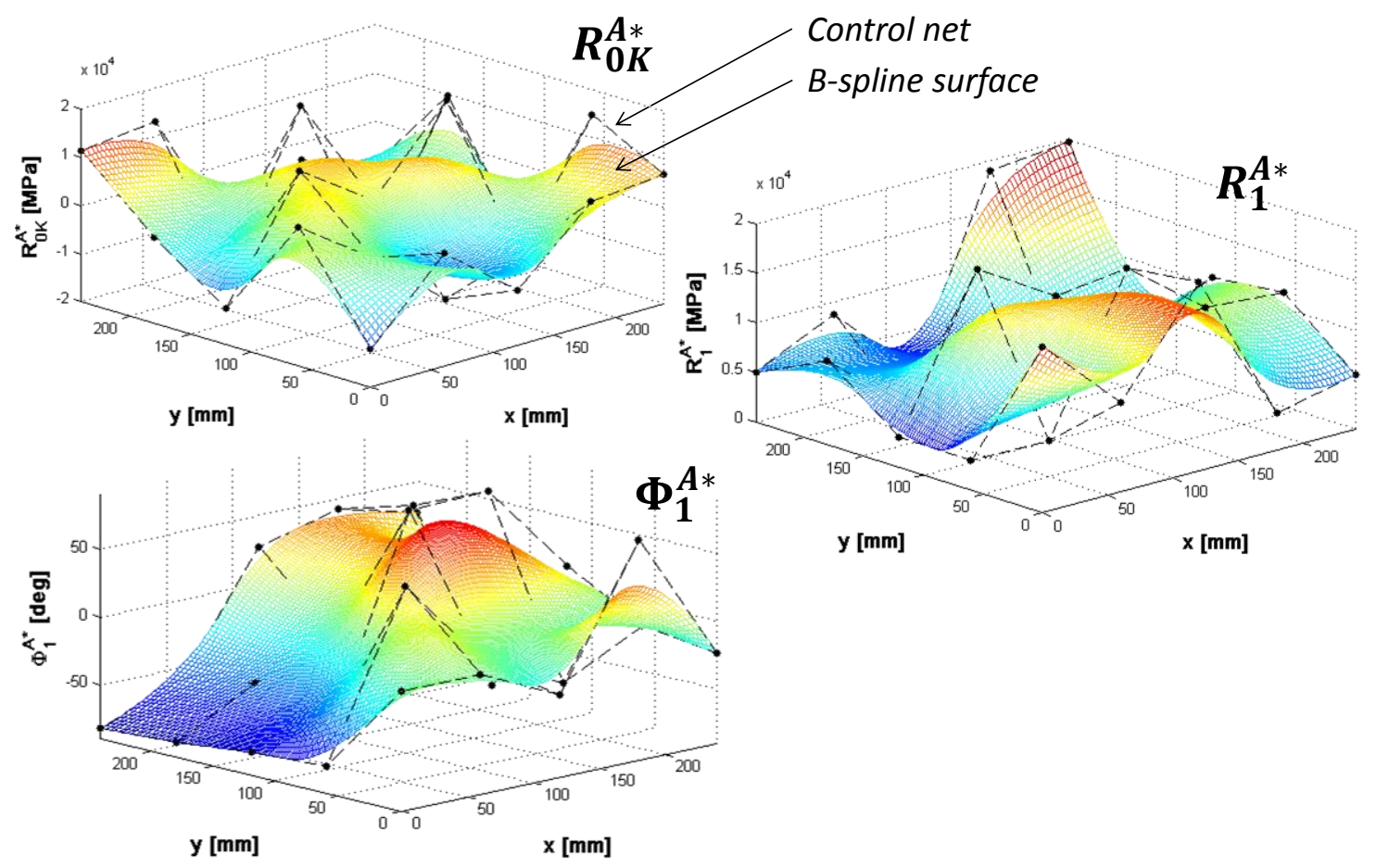

Figure 1: Example of B-spline surfaces in the space of the laminate polar parameters.

The dimensionless coordinates $\xi$ and $\gamma$ can be arbitrarily defined: a natural choice consists in linking them with the Cartesian coordinates of the laminated plate,

$$
\xi=\frac{x}{a}, \gamma=\frac{y}{b},
$$

where $a$ and $b$ are the lengths of the plate edges along $x$ and $y$ axes, respectively. In Eq. (4) $\left\{R_{0 K}^{A^{*}(i, j)}, R_{1}^{A^{*}(i, j)}, \Phi_{1}^{A^{*(i, j)}}\right\}\left(i=0, \cdots, n_{p}, j=0, \cdots, m_{p}\right)$ are the values of the laminate polar parameters at the generic control point (the set of $\left(n_{p}+1\right) \times\left(m_{p}+1\right)$ control points forms the so-called control network), while $N_{i, p}(\xi)$ and $N_{j, q}(\gamma)$ are the $p^{\text {th }}$-degree and $q^{\text {th }}$-degree B-spline basis functions (along $\xi$ and $\gamma$ directions, respectively) defined on the non-periodic, non-uniform knot vectors:

$$
\begin{aligned}
& \boldsymbol{\Xi}=\{\underbrace{0, \cdots, 0}_{p+1}, \Xi_{p+1}, \cdots, \Xi_{r-p-1}, \underbrace{1, \cdots, 1}_{p+1}\}, \\
& \boldsymbol{\Gamma}=\{\underbrace{0, \cdots, 0}_{q+1}, \Gamma_{q+1}, \cdots, \Gamma_{s-q-1}, \underbrace{1, \cdots, 1}_{q+1}\} .
\end{aligned}
$$

It is noteworthy that the dimensions of the knot-vectors $\boldsymbol{\Xi}$ and $\boldsymbol{\Gamma}$ are $r+1$ and $s+1$, 
respectively, with:

$$
\begin{aligned}
& r=n_{p}+p+1, \\
& s=m_{p}+q+1 .
\end{aligned}
$$

For a deeper insight in the matter the reader is addressed to [32].

As previously stated, the use of iso-geometric surfaces for describing the variation of the mechanical design variables over the structure, implies that the three independent polar parameters $\Phi_{1}^{A^{*}}, R_{0 K}^{A^{*}}$ and $R_{1}^{A^{*}}$ have no discontinuity over the plate. Moreover, thanks to the B-spline representation the mechanical design variables (i.e. the laminate polar parameters) must be determined solely on each point of the control net, implying in this way a significant reduction in the number of design variables involved within the first-level problem.

Therefore, the optimisation variables of the problem can be grouped into the following vector:

$$
\mathbf{x}=\left\{\Phi_{1}^{A^{*(0,0)}}, \cdots, \Phi_{1}^{A^{*}\left(n_{p}, m_{p}\right)}, R_{0 K}^{A^{*}(0,0)}, \cdots, R_{0 K}^{A^{*}\left(n_{p}, m_{p}\right)}, R_{1}^{A^{*}(0,0)}, \cdots, R_{1}^{A^{*}\left(n_{p}, m_{p}\right)}\right\} .
$$

The total number of design variables is hence equal to $3 \times\left(n_{p}+1\right) \times\left(m_{p}+1\right)$.

In addition, in the formulation of the optimisation problem for the first level of the strategy, the geometric and feasibility constraints on the polar parameters (which arise from the combination of the layer orientations and positions within the stack) must also be considered. These constraints ensure that the optimum values of the polar parameters resulting from the first step correspond to a feasible laminate that will be designed during the second step of the optimisation strategy, see [38]. Since the laminate is quasi-homogeneous, such constraints can be written only for matrix $\left[\mathrm{A}^{*}\right]$ as follows:

$$
\left\{\begin{array}{c}
-R_{0} \leq R_{0 K}^{A^{*}} \leq R_{0}, \\
0 \leq R_{1}^{A^{*}} \leq R_{1}, \\
2\left(\frac{R_{1}^{A^{*}}}{R_{1}}\right)^{2}-1-\frac{R_{0 K}^{A^{*}}}{R_{0}} \leq 0 .
\end{array}\right.
$$

As explained beforehand, thanks to the strong convex-hull property these constraints have to be checked only on the points of the control network. If they are met on these points they will be satisfied on the whole domain of the B-spline surface. This aspect represents a further advantage when using the B-spline representation for the mechanical design variables. Moreover, first and second constraints of Eq. (9) can be taken into account as admissible intervals for the relevant optimisation variables, i.e. on $R_{0 K}^{A^{*}(i, j)}$ and $R_{1}^{A^{*}(i, j)}$. Hence, the resulting feasibility constraint on the laminate polar parameters of the generic control point is:

$$
g_{i j}(\mathbf{x})=2\left(\frac{R_{1}^{A^{*}(i, j)}}{R_{1}}\right)^{2}-1-\frac{R_{0 K}^{A^{*}(i, j)}}{R_{0}} \leq 0
$$


with $i=0, \cdots, n_{p}$ and $j=0, \cdots, m_{p}$. The total number of feasibility constraints to be imposed is thus equal to $\left(n_{p}+1\right) \times\left(m_{p}+1\right)$.

For a wide discussion upon the laminate feasibility and geometrical bounds as well as on the importance of the quasi-homogeneity assumption the reader is addressed to [38].

\subsection{Mathematical statement of the problem}

The first-level problem focuses on the definition of the optimal distribution of the laminate polar parameters [6]. In this background, the solution of the structural optimisation problem is searched for an orthotropic uncoupled homogenised (locally, i.e. point-wise) plate subject to given BCs.

Therefore the optimisation problem can be formulated as follows:

$$
\begin{aligned}
& \min _{\mathbf{x}}-\lambda(\mathbf{x}) \\
& \text { subject to: } \\
& g_{i j}(\mathbf{x}) \leq 0,\left(i=0, \cdots, n_{p}, \quad j=0, \cdots, m_{p}\right)
\end{aligned}
$$

where $\lambda$ is the first buckling factor of the laminated structure.

\subsection{Numerical strategy}

Problem (11) is a non-linear, non-convex problem in terms of the mechanical design variables. Its non-linearity and non-convexity is due to the nature of the objective function, the first buckling factor, that is a non-convex function in terms of the orthotropy orientation. In addition, the complexity of such a problem is also due to the feasibility constraints imposed on the polar parameters of the plate, see Eq. (10). We recall that the overall number of design variables and optimisation constraints for problem (11) is $3 \times\left(n_{p}+1\right) \times\left(m_{p}+1\right)$ and $\left(n_{p}+1\right) \times\left(m_{p}+1\right)$, respectively.

For the resolution of problem (11) a hybrid optimisation tool, composed of the GA BIANCA [17] interfaced with the MATLAB fmincon algorithm [16], coupled with a FE model of the plate (used for numerical calculation of the first buckling load) has been developed, see Fig. 2.

The GA BIANCA was already successfully applied to solve different kinds of realworld engineering problems, see for example [5, 25]. As shown in Fig. 2, the optimisation procedure for the first-level problem is split in two phases. During the first phase the GA BIANCA is interfaced with the FE model of the VAT plate: for each individual at each generation, a FE-based buckling analysis is carried out for the evaluation of the first buckling load of the structure. The FE model makes use of the mechanical design variables, given by BIANCA and elaborated by an ANSYS Parametric Design Language (APDL) macro which generates the B-spline surface representing the distribution of the polar parameters over the VAT plate, in order to calculate the first buckling load of the 


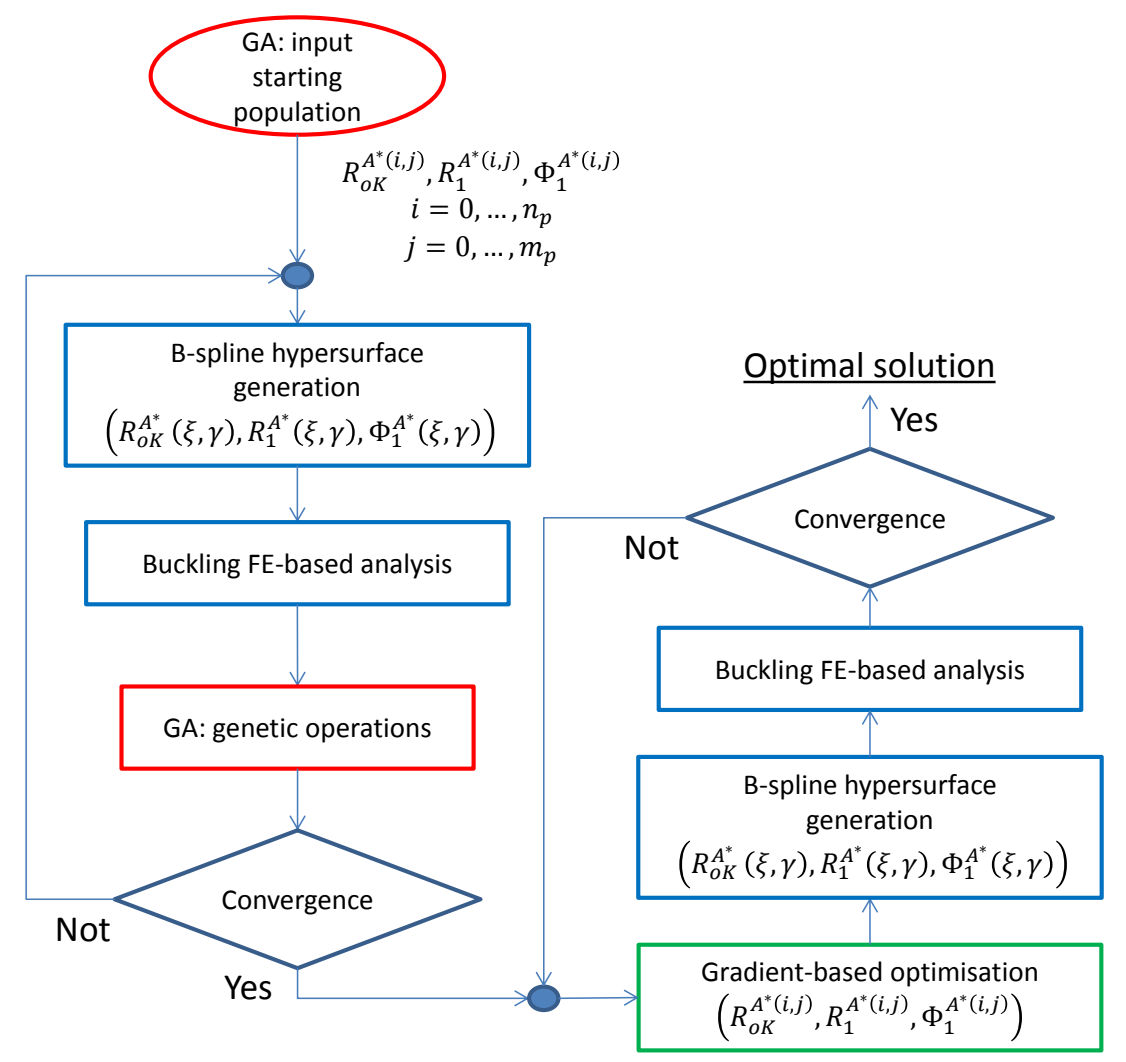

Figure 2: Logical flow of the numerical procedure for the solution search of the first-level problem.

structure. Therefore, for these purposes the GA (coded in Fortran environment) has been interfaced with the commercial FE code ANSYS. At the end of the FE analysis, the GA elaborates the results provided by the FE model (in terms of objective and constraint functions) in order to execute the genetic operations. These operations are repeated until the GA meets the user-defined convergence criterion. The generic individual of the GA represents a potential solution for the problem at hand. The genotype of the individual for problem (11) is characterised by $\left(n_{p}+1\right) \times\left(m_{p}+1\right)$ chromosomes composed of 3 genes, each one coding a component of the vector of the design variables. Due to the strong nonconvex nature of problem (11), the aim of the genetic calculation is to provide a potential sub-optimal point in the design space which constitutes the initial guess for the subsequent phase, i.e. the local optimisation, where the fmincon gradient-based algorithm is interfaced with the same FE model of the VAT plate.

\section{Mathematical formulation of the second-level problem}

The second-level problem concerns the lay-up design of the VAT laminated plate. The goal of this problem is the determination of at least one stacking sequence satisfying the 
optimum values of the distribution of the polar parameters over the structure resulting from the first level of the strategy and having the elastic symmetries imposed to the laminate within the formulation of the first-level problem, i.e. quasi-homogeneity and orthotropy.

In the case of a VAT solution the fibres orientation angle varies point-wise in every ply composing the laminate. Hence a proper description of the fibres path is necessary to formulate and solve the second-level problem of the MS2L strategy. To this purpose, the following modifications have been brought to the second step of the MS2L optimisation procedure:

- the point-wise variation of the fibre orientation (in each ply) is described through the use of a B-spline surface;

- the technological constraint on the minimum radius of curvature of the pre-preg strips is taken into account.

These improvements to the second step of the strategy lead to important advantages in solving the related optimisation problem. In fact, the use of B-spline surfaces allows, as in the case of the first-level problem, to reduce the total number of design variables: in this case it is sufficient to calculate the fibre orientation solely at each point of the B-spline control network. In addition, thanks to the use of iso-geometric blending functions the local steering (i.e. the local radius of curvature of the tow) can be determined easily and introduced in the problem formulation as an optimisation constraint. This last aspect is of paramount importance to obtain a proper formulation of the technological constraints regarding the layout of pre-preg strips in each ply which cannot exceed a given curvature.

Concerning the representation of the fibres path, the relative B-spline surface for each ply is defined as:

$$
\delta_{k}(\xi, \gamma)=\sum_{i=0}^{n_{p}} \sum_{j=0}^{m_{p}} N_{i, p}(\xi) N_{j, q}(\gamma) \delta_{k}^{(i, j)} \text { with } k=1, \cdots, n
$$

In this case $\delta_{k}{ }^{(i, j)}$ is the orientation angle at the generic control point for the $\mathrm{k}$-th layer, i.e. the design variables of the second-level problem of the MS2L strategy whose overall number is equal to $n \times\left(n_{p}+1\right) \times\left(m_{p}+1\right)$.

In the framework of the polar formalism, the problem of the lay-up design of the VAT laminate can be stated in the form of a constrained minimisation problem:

$$
\left\{\begin{array}{cl}
\min _{\delta_{k}(i, j)} I\left(\delta_{k}^{(i, j)}\right) \quad & k=1, \cdots, n, \\
& i=0, \cdots, n_{p}, \\
& j=0, \cdots, m_{p}, \\
r_{a d m}-r_{\min } \leq 0 . &
\end{array}\right.
$$

In Eq. (13) $r_{a d m}$ is the minimum admissible radius of curvature of the tow whose value depends upon the AFP process, while $r_{\min }$ is the local least radius of curvature among all 
the plies. $r_{\min }$ is defined as:

$$
\begin{array}{ll}
r_{\text {min }}=\min _{k}\left[\min _{(x, y)} r_{k}(x, y)\right], & \\
r_{k}(x, y)=\left(\mathbf{t}_{k} \cdot \nabla \delta_{k}\right)^{-1}, & k=1, \cdots, n, \\
& x \in[0, a], \\
y & \in[0, b] .
\end{array}
$$

In Eq. (14) $\mathbf{t}_{k}$ is the local tangent vector of the angular field $\delta_{k}(x, y)$ of the $k$-th ply, while $\nabla \delta_{k}$ is the gradient of the fibre path with respect to coordinates $(x, y)$, namely

$$
\begin{aligned}
\mathbf{t}_{k} & =\left\{\cos \delta_{k}, \sin \delta_{k}\right\}, \\
\nabla \delta_{k} & =\left\{\frac{1}{a} \frac{\partial \delta_{k}}{\partial \xi}, \frac{1}{b} \frac{\partial \delta_{k}}{\partial \gamma}\right\} .
\end{aligned}
$$

In Eq. (13) $I\left(\delta_{k}{ }^{(i, j)}\right)$ is the overall objective function which is defined as:

$$
I\left(\delta_{k}^{(i, j)}\right)=\sum_{i=1}^{6} f_{i}\left(\delta_{k}^{(i, j)}\right) .
$$

where $f_{i}\left(\delta_{k}^{(i, j)}\right)$ are quadratic functions in the space of polar parameters, each one representing a requirement to be satisfied. For the problem at hand the partial objective functions write:

$$
\begin{gathered}
f_{1}\left(\delta_{k}^{(i, j)}\right)=\int_{0}^{1} \int_{0}^{1}\left[\frac{\Phi_{0}^{A^{*}}\left(\delta_{k}(\xi, \gamma)\right)-\Phi_{1}^{A^{*}}\left(\delta_{k}(\xi, \gamma)\right)}{\pi / 4}-K^{A^{*}(o p t)}(\xi, \gamma)\right]^{2} d \xi d \gamma \\
f_{2}\left(\delta_{k}^{(i, j)}\right)=\int_{0}^{1} \int_{0}^{1}\left[\frac{R_{0}^{A^{*}}\left(\delta_{k}(\xi, \gamma)\right)-R_{0}^{A^{*}(o p t)}(\xi, \gamma)}{R_{0}}\right]^{2} d \xi d \gamma \\
f_{3}\left(\delta_{k}^{(i, j)}\right)=\int_{0}^{1} \int_{0}^{1}\left[\frac{R_{1}^{A^{*}}\left(\delta_{k}(\xi, \gamma)\right)-R_{1}^{A^{*}(o p t)}(\xi, \gamma)}{R_{1}}\right]^{2} d \xi d \gamma, \\
f_{4}\left(\delta_{k}^{(i, j)}\right)=\int_{0}^{1} \int_{0}^{1}\left[\frac{\Phi_{1}^{A^{*}}\left(\delta_{k}(\xi, \gamma)\right)-\Phi_{1}^{A^{*}(o p t)}(\xi, \gamma)}{\pi / 4}\right]^{2} d \xi d \gamma \\
f_{5}\left(\delta_{k}^{(i, j)}\right)=\int_{0}^{1} \int_{0}^{1}\left[\frac{\left\|[\mathrm{C}]\left(\delta_{k}(\xi, \gamma)\right)\right\|}{\|[\mathrm{Q}]\|}\right]^{2} d \xi d \gamma \\
f_{6}\left(\delta_{k}^{(i, j)}\right)=\int_{0}^{1} \int_{0}^{1}\left[\frac{\left\|\left[\mathrm{B}^{*}\right]\left(\delta_{k}(\xi, \gamma)\right)\right\|}{\|[\mathrm{Q}]\|}\right]^{2} d \xi d \gamma,
\end{gathered}
$$

where

$$
K^{A^{*}(o p t)}(\xi, \gamma)=\left\{\begin{array}{ll}
1 & \text { if } \\
0 & \text { otherwise } .
\end{array} R_{0 K}^{A^{*}(o p t)}(\xi, \gamma)<0,\right.
$$

In Eq. (17) $f_{1}\left(\delta_{k}^{(i, j)}\right)$ represents the elastic requirement on the orthotropy of the laminate having the prescribed shape, $f_{2}\left(\delta_{k}^{(i, j)}\right), f_{3}\left(\delta_{k}^{(i, j)}\right)$ and $f_{4}\left(\delta_{k}^{(i, j)}\right)$ are the requirements related to the prescribed values of the optimal polar parameters resulting from the first-level problem, while $f_{5}\left(\delta_{k}^{(i, j)}\right)$ and $f_{6}\left(\delta_{k}^{(i, j)}\right)$ are linked to the quasi-homogeneity condition. For more details on the meaning of the partial objective functions, on the elastic symmetries of the 
laminate in the framework of the FSDT and on the symbols appearing in Eq. (17), the reader is addressed to $[22,23]$.

$I\left(\delta_{k}^{(i, j)}\right)$ is a positive semi-definite convex function in the space of laminate polar parameters, since it is defined as a sum of convex functions, see Eqs. (16)-(17). Nevertheless, such a function is highly non-convex in the space of plies orientations because the laminate polar parameters depend upon circular functions of the layers orientation angles, see $[22,23]$. Moreover, one of the advantages of such a formulation consists in the fact that the absolute minima of $I\left(\delta_{k}^{(i, j)}\right)$ are known a priori since they are the zeroes of this function. For more details about the nature of the second-level problem see [17, 18, 20]. Concerning the numerical strategy for solving problem (13) the GA BIANCA has been employed to find a solution also for the second-level problem. In this case, each individual is composed of $n$ chromosomes (one for each ply), each one characterised by $\left(n_{p}+1\right) \times\left(m_{p}+1\right)$ genes coding the layer orientation angle for each control point of the chromosome-ply.

\section{$5 \quad$ Finite element model of the VAT laminate}

In order to determine the current value of the objective function (the first buckling factor) and that of the optimisation constraints of problem (11) a linear buckling analysis must be achieved for the VAT composite. The need to analyse, within the same calculation, different configurations of the VAT plate requires the creation of an ad-hoc input file for the FE model that has to be interfaced with the hybrid (GA + gradient-based algorithms) optimisation tool.

The FE model of the VAT laminated plate (see Fig. 3) employed during the first step of the MS2L strategy, is built within the ANSYS environment and is made of SHELL281 elements based on the Reissner-Mindlin kinematic model, having 8 nodes and six Degrees Of Freedom (DOFs) per node. The mesh size is chosen after preliminary mesh sensitivity analyses on the convergence of the value of the first buckling load for a given set of BCs. It was observed that a mesh having 2482 DOFs is sufficient to properly evaluate the first buckling load of the structure.

It is noteworthy that the B-Spline mathematical formalism has been implemented by the authors into the ANSYS environment by using the ANSYS APDL [2] for creating a set of appropriate macros that were integrated within the FE model of the VAT plate. At this stage, the plate is modelled as an equivalent homogeneous anisotropic plate whose stiffness matrices $\left(\left[\mathrm{A}^{*}\right],\left[\mathrm{B}^{*}\right],\left[\mathrm{D}^{*}\right]\right.$ and $\left.\left[\mathrm{H}^{*}\right]\right)$ vary point-wise, i.e. for each element discretising the real structure. In particular, in order to properly define, for every element of the VAT plate, the correct value of its stiffness properties the following strategy has been employed:

1. for a given set of the laminate polar parameters defined in each control point (the design variables passed from the optimisation tool to the FE model of the VAT plate, 

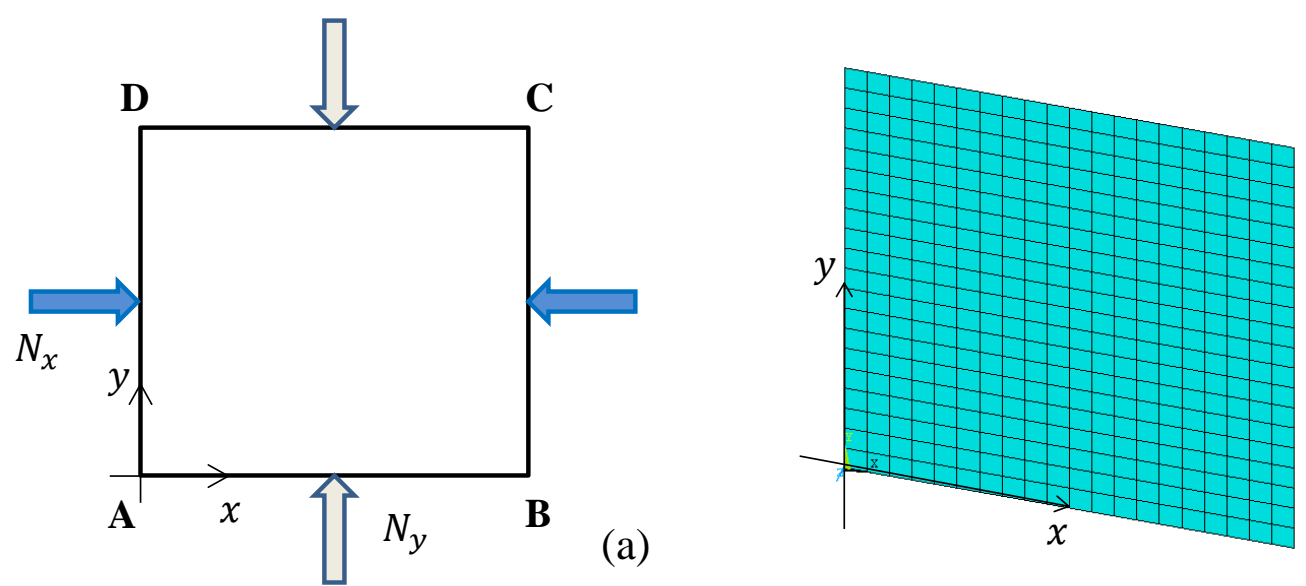

(b)

Figure 3: Geometry of the VAT plate and applied BCs (a) and FE model of the structure (b).

see Fig. 3), build the corresponding B-spline surfaces;

2. discretise the plate into $N_{e}$ elements;

3. fix the element index $i$ : for the $i$-th element retrieve the Cartesian coordinates of its centroid, i.e. $\left(x_{e}^{i}, y_{e}^{i}\right)$ and calculate the corresponding dimensionless coordinates $\left(\xi_{e}^{i}, \gamma_{e}^{i}\right)$ according to Eq. (5);

4. calculate the laminate polar parameters (and hence the Cartesian components of the stiffness matrices of the laminate) for $\left(\xi_{e}^{i}, \gamma_{e}^{i}\right)$ and assign the material properties to the element $i$;

5. repeat points 3 and 4 for each element of the plate.

Finally, the linear buckling analysis is performed using the BCs depicted in Fig. 3 and listed in Table 2.

Sides BCs

\begin{tabular}{ll}
\hline $\mathrm{AB}, \mathrm{CD}$ & $U_{x}=0$ \\
& $U_{z}=0$ \\
$\mathrm{BC}, \mathrm{DA}$ & $U_{y}=0$ \\
& $U_{z}=0$ \\
\hline
\end{tabular}

Table 2: BCs of the FE model of the VAT laminated plate. 


\section{Numerical example}

In this section a meaningful numerical example is considered in order to prove the effectiveness of the MS2L strategy for the optimum design of VAT laminates. As depicted in Fig. 3, a bi-axial compressive load per unit length is applied on the plate edges with a ratio $\frac{N_{y}}{N_{x}}=0.5$. The plate has a square geometry with side length $a=b=254 \mathrm{~mm}$ and is made of $n=24$ plies whose material properties are those listed in Table 1. Concerning the first-level problem, the parameters defining the B-spline surfaces which describe the polar parameters distribution over the VAT plate are set as: $n_{p}=m_{p}=4$ (hence five control points along each direction), $p=q=2$ (degrees of the blending functions along each direction). Moreover, each B-spline is defined over the following uniform knot-vectors:

$$
\begin{aligned}
& \boldsymbol{\Xi}=\left\{0,0,0, \frac{1}{3}, \frac{2}{3}, 1,1,1\right\}, \\
& \boldsymbol{\Gamma}=\left\{0,0,0, \frac{1}{3}, \frac{2}{3}, 1,1,1\right\} .
\end{aligned}
$$

Accordingly, for the first-level problem the overall number of design variables and optimisation constraints is 75 and 25, respectively. The mechanical design variables together with their nature and bounds for the first-level problem are listed in Table 3.

\begin{tabular}{lllll}
\hline $\begin{array}{l}\text { Design } \\
\text { able }\end{array}$ & vari- & Type & Lower bound & Upper bound \\
\hline$R_{0 K}^{A *}(i, j)$ & {$[\mathrm{MPa}]$} & continuous & -17693.3868 & 17693.3868 \\
$R_{1}^{A *}{ }^{(i, j)}[\mathrm{MPa}]$ & continuous & 0.0 & 19072.0711 \\
$\Phi_{1}^{A *(i, j)}[\mathrm{deg}]$ & continuous & -90.0 & 90.0 \\
\hline
\end{tabular}

Table 3: Design space of the first-level problem.

Concerning the second-level problem, the parameters defining the B-spline surface which describes the point-wise variation of the fibre orientation angle (for each ply) are the same as those employed during the first step of the strategy. This means that the overall number of design variables for the second-level problem is significant and equal to 600 (i.e. 25 orientation angles defined in each control point per layer), while there is only one optimisation constraint, see Eq. (13). In addition, the reference value for the minimum admissible radius of curvature of the tow, i.e. $r_{a d m}$ is set equal to $30 \mathrm{~mm}$.

It must be highlighted the fact that $\delta_{k}^{(i, j)}$ are continuous variables in the range $\left[-90^{\circ}\right.$, $\left.90^{\circ}\right]$.

Regarding the setting of the genetic parameters for the GA BIANCA utilised for both first and second-level problems they are listed in Table 4. Moreover, concerning the constraint-handling technique for both levels of the strategy the Automatic Dynamic Penalization (ADP) method has been employed, see [21]. For more details on the numerical techniques developed within the new version of BIANCA and the meaning of the values of the different parameters tuning the GA the reader is addressed to [17, 20]. 


\begin{tabular}{|c|c|c|}
\hline \multicolumn{3}{|c|}{ Genetic parameters } \\
\hline & $\begin{array}{l}1^{\text {st }} \text { level prob- } \\
\text { lem }\end{array}$ & $\begin{array}{l}2^{\text {nd }} \text { level prob- } \\
\text { lem }\end{array}$ \\
\hline N. of populations & 1 & 1 \\
\hline N. of individuals & 500 & 2000 \\
\hline N. of generations & 200 & 1000 \\
\hline $\begin{array}{l}\text { Crossover proba- } \\
\text { bility }\end{array}$ & 0.85 & 0.85 \\
\hline $\begin{array}{l}\text { Mutation proba- } \\
\text { bility }\end{array}$ & 0.002 & 0.0005 \\
\hline $\begin{array}{l}\text { Selection operator } \\
\text { Elitism operator }\end{array}$ & $\begin{array}{l}\text { roulette-wheel } \\
\text { active }\end{array}$ & $\begin{array}{l}\text { roulette-wheel } \\
\text { active }\end{array}$ \\
\hline
\end{tabular}

Table 4: Genetic parameters of the GA BIANCA for both first and second-level problems.

As far as concerns the fmincon optimisation tool employed for the local solution search at the end of the first step, the numerical algorithm chosen to carry out the calculations is the active-set method with non-linear constraints. For more details on the gradient-based approaches implemented into MATLAB, the reader is addressed to [16].

Before starting the multi-scale optimisation process a reference structure must be defined in order to establish a reference value for the first buckling factor of the plate. The reference structure is still a square plate of side $a=b=254 \mathrm{~mm}$ composed of 24 unidirectional fibre-reinforced laminae whose material properties are those listed in Table 1 . The stacking sequence of the reference solution is $[0 /-45 / 0 / 45 / 90 / 45 / 0 /-45 / 90 / 45 / 90 /-45]_{s}$. The choice of the reference solution has been oriented towards a symmetric quasi-isotropic stack, of common use in real-world engineering applications, which constitutes a "good" compromise between weight and stiffness requirements (in terms of buckling load): such a configuration is characterised by a buckling factor $\lambda_{r e f}=81.525$ when $N_{x}=1 \mathrm{~N} / \mathrm{mm}$ and $N_{y}=0.5 \mathrm{~N} / \mathrm{mm}$.

Concerning the first-level problem, the optimum distribution of the laminate polar parameters over the VAT plate is illustrated in Fig. 4, while the optimum value of the mechanical design variables for each control point are listed in Tables $5-7$. On the other hand, concerning the solution of the second-level problem, an illustration of the optimum fibres-path for the firsts four layers (for sake of synthesis) is depicted in Fig. 5 . It is noteworthy that the optimal solution found at the end of the MS2L design procedure is characterised by a buckling factor of 173.94 which is about $114 \%$ higher than the reference counterpart and, in the meantime, satisfies the technological constraint on the minimum (local) radius of curvature of the tow imposed by the AFP process.

From a careful analysis of the optimum configuration of the VAT laminated plate provided by the MS2L procedure, it is possible to deduce the following facts. 

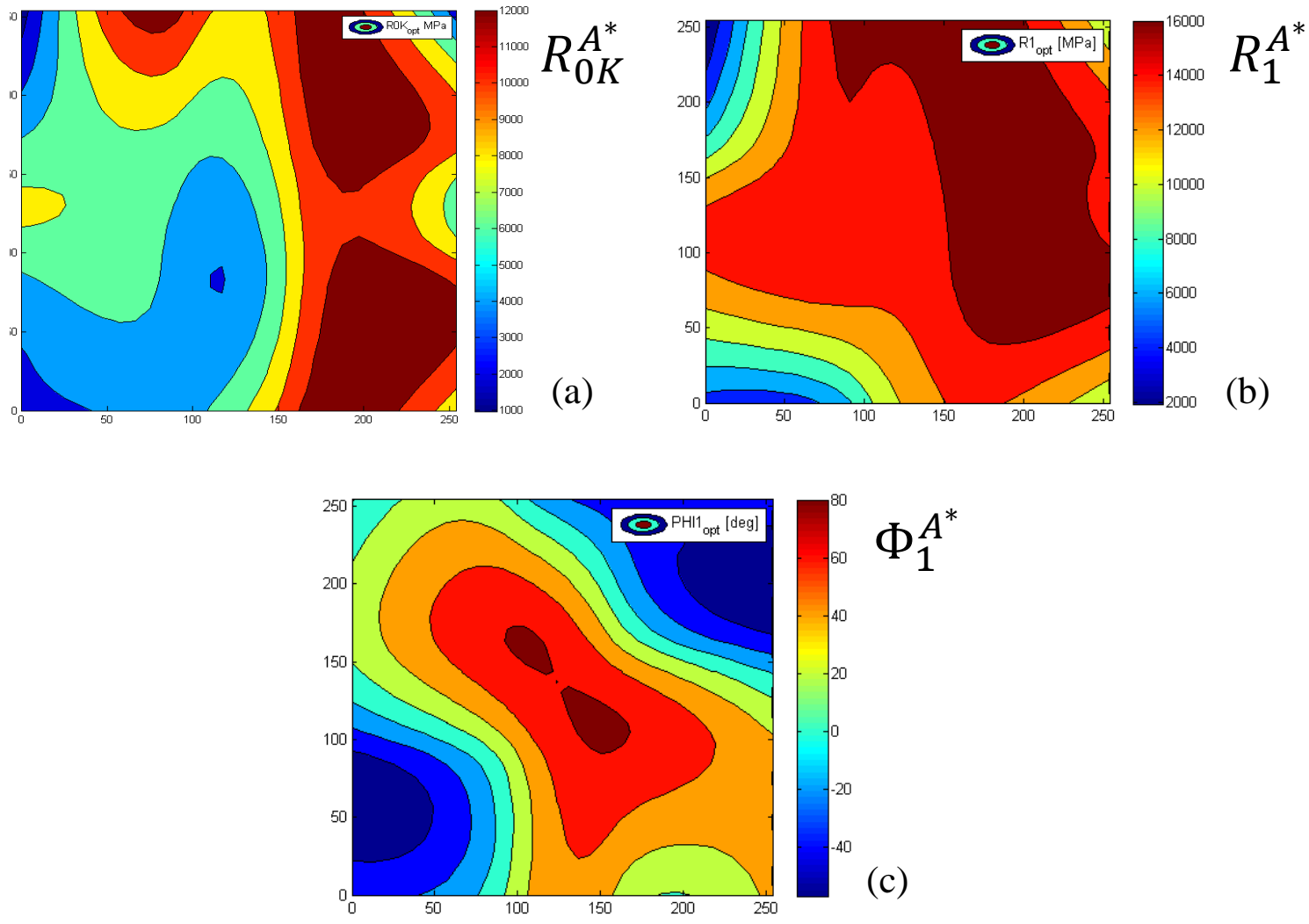

Figure 4: Optimal distribution of the polar parameters $R_{0 K} A^{*}$ (a), $R_{1}{ }^{*}$ (b) and $\Phi_{1} A^{*}$ (c) over the VAT plate resulting from the first-level optimisation problem.

- The polar parameters distribution resulting from the first step of the strategy is totally asymmetric. Symmetric solutions are, of course, possible: it is sufficient to impose the symmetry condition directly on the values of the laminate polar parameters in the points of the control network of the B-spline surfaces. However, in order to state and solve the optimisation problem in the most general case, in this study we prefer of not imposing such a condition. Indeed, when problem (11) is formulated and solved in the most general case, it is possible to find non-conventional asymmetric distributions of the mechanical design variables characterised by significantly superior performances when compared to classical symmetric, quasi-isotropic stacks often employed in the aeronautical field.

- When looking at the optimum distribution of the laminate polar parameters (Fig. 4), one can notice that the laminate is always characterised by an ordinary orthotropy shape with $K^{A^{*}}=0$ because $R_{0 K}^{A^{*}}(\xi, \gamma)$ is strictly positive over the laminated plate.

- Unlike the vast majority of works reported in literature [40], the optimum fibres-path for each ply is very general. In the framework of the proposed approach, the pointwise variation of the fibre orientation angle in every lamina does not follow simple 


\begin{tabular}{llllll}
\hline \multicolumn{1}{r}{$m_{p}$} & 0 & 1 & 2 & 3 & 4 \\
\hline 0 & & & & & \\
1 & 3591.3747 & 3668.8094 & 9592.3869 & 4497.2954 & 975.9237 \\
2 & 4004.9094 & 8579.8092 & 7560.9021 & 9286.1572 & 17011.3438 \\
3 & 6579.9750 & 805.5577 & 3036.0234 & 5777.9895 & 6901.0129 \\
4 & 16467.7981 & 16448.7345 & 15040.6238 & 16596.7375 & 16926.6619 \\
& 8529.0554 & 15762.0163 & 4406.7537 & 14321.3969 & 2829.6760 \\
\hline
\end{tabular}

Table 5: Optimum value of $R_{0 K} A^{*}$ [MPa] for each control point of the B-spline surface.

\begin{tabular}{|c|c|c|c|c|c|}
\hline$n_{p}$ & 0 & 1 & 2 & 3 & 4 \\
\hline 0 & 5205.1699 & 12784.5159 & 16065.5107 & 5068.0708 & 1932.6002 \\
\hline 1 & 3447.8699 & 16298.3907 & 16011.8612 & 16593.1744 & 17332.2704 \\
\hline 2 & 13511.7905 & 13789.0773 & 14452.1311 & 15045.4781 & 15809.2434 \\
\hline 3 & 14966.4897 & 18733.6564 & 18342.5388 & 18722.0860 & 18735.1483 \\
\hline 4 & 10265.5066 & 17842.4603 & 14882.8151 & 16217.0759 & 10244.8019 \\
\hline
\end{tabular}

Table 6: Optimum value of $R_{1}{ }^{*}$ [MPa] for each control point of the B-spline surface.

linear or parabolic variations (with respect to laminate global frame) as in [40], rather it is described by a general B-spline surface, see Eq. (12). This fact, together with the very general formulation of problem (13), allows the designer to find (at the cost of a considerable computation effort) an optimum stack meeting all the requirements (i.e. elastic and manufacturability constraints) provided by problem (13), without the need of a further post-processing treatment to simplify the trajectory of the tows in order to comply with the constraints imposed by the AFP process.

- Finally, the optimum fibres-path (for each layer) found at the end of the second step of the MS2L procedure does not need of a further step for the reconstruction of the CAD model because the variation of the fibres-path is described by a B-spline surface which is fully compatible with several standard file formats (IGES, STL and STEP),

\begin{tabular}{|c|c|c|c|c|c|}
\hline$n_{p}$ & 0 & 1 & 2 & 3 & 4 \\
\hline 0 & -20.9816 & -84.5402 & 11.1669 & 38.4351 & -0.9851 \\
\hline 1 & -30.7624 & -80.1076 & 56.6921 & 86.1616 & 53.2841 \\
\hline 2 & 79.6062 & 82.6653 & 89.9998 & 88.5717 & -26.4919 \\
\hline 3 & -3.9816 & 60.5155 & 89.3887 & -88.5577 & -28.6826 \\
\hline 4 & 45.3655 & 57.9974 & 30.2596 & -84.8941 & -26.7837 \\
\hline
\end{tabular}

Table 7: Optimum value of $\Phi_{1}{ }^{*}$ [deg] for each control point of the B-spline surface. 


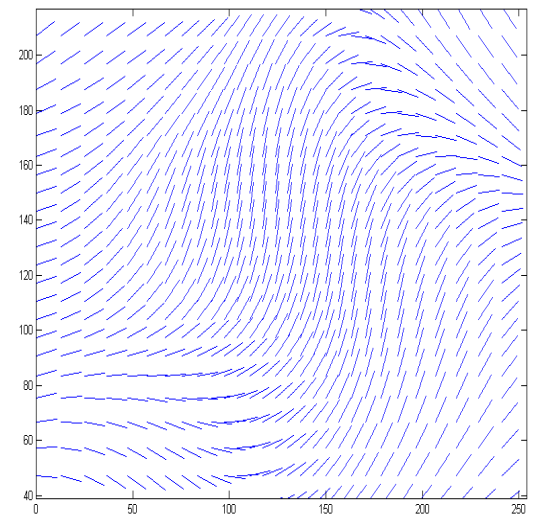

(a)

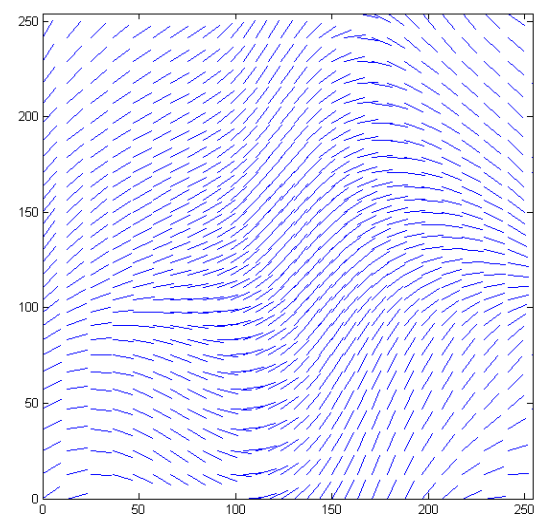

(b)

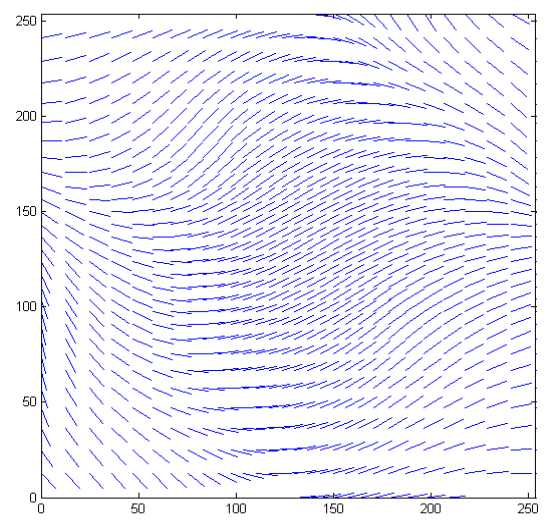

(c)

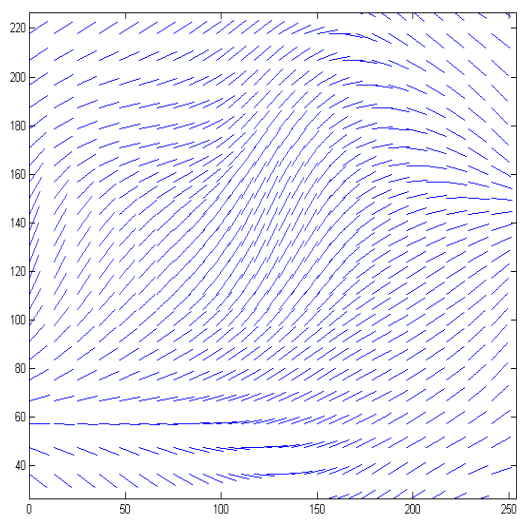

(d)

Figure 5: Optimum fibres path for the firsts four layers of the VAT plate resulting from the second-level optimisation problem, $1^{\text {st }}$ ply (a), $2^{\text {nd }}$ ply (b), $3^{\text {rd }}$ ply (c) and $4^{\text {th }}$ ply (d).

allows in this way a rapid exchange of information among the CAD tool and the software of the AFP process.

\section{Conclusions and perspectives}

In this work a new paradigm for the design and optimisation of VAT composite structures is presented. This paradigm essentially relies on the utilisation of a MS2L optimisation procedure characterised by several features that make it an original, effective and general method for the multi-scale design of complex VAT structures. In the present work this strategy has been employed to deal with the problem of the maximisation of the buckling factor of a VAT plate subject to both mechanical and manufacturability constraints. On the one hand, the design process is not submitted to restrictions: any parameter characterising the VAT composite (at each scale) is an optimisation variable. This allows the designer to look for a true global minimum, hard to be obtained otherwise. On the other hand, both the formulation of the design problem and the MS2L optimisation strategy have been generalised and improved in order to be applied to the problem of designing a VS composite. 
In the framework of the MS2L design methodology several modifications have been introduced for both first and second level problems.

Concerning the first-level problem the main modifications are: 1) the use of higherorder theories (introduced as result of $[22,23,24]$ ) for taking into account the influence of the transverse shear stiffness on the overall mechanical response of VAT composites and 2) the utilisation of B-spline surfaces for describing the distribution of the laminate polar parameters over the structure which allow for a continuous point-wise variation of the laminate stiffness matrices. This last aspect leads to some important advantages for the resolution of the related optimisation problem. Firstly, the utilisation of B-spline surfaces leads to a considerable reduction in the number of design variables (the polar parameters have to be defined solely in each point of the control network of the B-spline surface). Secondly, thanks to the strong convex-hull property of the B-spline blending functions the optimisation constraints of the problem (related to the specifications of the considered application) can be imposed only on the control points of the network: if they are satisfied on such points they are automatically met over the whole domain.

For the second-level problem the major modifications are: 1) the utilisation of B-spline basis functions for obtaining a continuous point-wise variation of the fibre orientation angle within each ply; 2) a proper mathematical formalisation of the manufacturability constraints linked to the AFP process in the framework of the B-spline representation. Also in the second step of the procedure, these modifications imply some important consequences. On the one hand the utilisation of B-spline surfaces leads to an important reduction of the number of design variables (the orientation angles defined in each control point of the layer), while on the other hand the B-spline mathematical formalism allows to express in a closed analytical form the manufacturability constraints linked to the AFP process. All of these modifications allow to go beyond the main restrictions characterising the design activities and research studies on VAT composites that one can find in literature.

Finally, the improved version of the MS2L strategy has been tested through a meaningful numerical example which proved its effectiveness. The optimisation tool gives an optimum VAT laminate characterised by a significant increment of the first buckling factor (about the 114\%) when compared to a reference classical solution (composed of unidirectional plies).

Concerning the perspectives of this work, there are still some theoretical, numerical and technical aspects and features that need to be deeply investigated and developed in order to make the proposed approach a very general and comprehensive strategy able to provide solutions that are both efficient (true optimal configurations) and manufacturable. Of course, this action passes through a real understanding of the potential and the technological restrictions linked to the AFP process. Currently, only the technological constraint on the tow steering has been integrated in the MS2L strategy. A step forward can be realised 
by properly formalising and including into the design problem other kinds of manufacturability constraints: tow gap and overlapping, the variation of the fibre volume fraction due to imperfections, etc. Moreover, in the framework of the MS2L optimisation procedure proposed in this work the manufacturability constraint linked to the minimum admissible radius of curvature of the tow has been integrated only within the second step of the design procedure. Actually, when using such an approach, there is no warranty that the optimisation algorithm could find an optimum fibres-path able to meet on the one hand the optimum distribution of the laminate polar parameters resulting from the first step of the strategy and on the other hand the manufacturability constraint related to the tow steering condition. To overcome such an issue, the formulation of the first-level problem should be modified accordingly, i.e. by integrating the manufacturability constraints since the first stage of the MS2L strategy. In addition, from a numerical point of view, the designer could be interested in optimising also the number of design variables (i.e. the number of the parameters tuning the shape of the B-spline surfaces) involved into both levels of the MS2L procedure: this point can be easily taken into account by exploiting the original features of the GA BIANCA. Finally, further modifications may also be considered in the formulation of the design problem depending on the nature of the considered application, e.g. by including constraints on inter- and intra-laminar damage, variability effects linked to the fabrication process, costs, etc.

Research is ongoing on all of the previous aspects.

\section{References}

[1] Abdalla MM, Setoodeh S, Gürdal Z (2007) Design of variable stiffness composite panels for maximum fundamental frequency using lamination parameters. Compos Struct 81:283-291

[2] Ansys (2013) ANSYS Mechanical APDL Basic Analysis Guide. Release 15.0, ANSYS, Inc., Southpointe, 275 Technology Drive, Canonsburg, PA 15317

[3] Blom AW, Abdalla MM, Gürdal Z (2010) Optimization of course locations in fiberplaced panels for general fiber angle distributions. Compos Sci Tec 70:564-570

[4] Catapano A, Montemurro M (2014) A multi-scale approach for the optimum design of sandwich plates with honeycomb core. Part I: homogenisation of core properties. Compos Struct 118:664-676

[5] Catapano A, Montemurro M (2014) A multi-scale approach for the optimum design of sandwich plates with honeycomb core. Part II: the optimisation strategy. Compos Struct 118:677-690 
[6] Catapano A, Desmorat B, Vannucci A (2015) Stiffness and strength optimization of the anisotropy distribution for laminated structures. J Optimiz Theory App 167(1):118-146

[7] Dang TD, Hallet SR (2013) A numerical study on impact and compression after impact behaviour of variable angle tow laminates. Compos Struct 96:194-206

[8] Ghiasi H, Pasini D, Lessard L (2009) Optimum stacking sequence design of composite materials. Part I : Constant stiffness design. Compos Struct 90:1-11

[9] Ghiasi H, Fayazbakhsh K, Pasini D, Lessard L (2010) Optimum stacking sequence design of composite materials. Part II : Variable stiffness design. Compos Struct 93:1-13

[10] Gürdal Z, Tatting BF, Wu KC (2008) Variable stiffness panels: Effects of stiffness variation on the in-plane and buckling responses. Compos Part A-Appl S 39(9):11-22

[11] Hammer VB, Bendsoe MP, Lipton R, Pedersen P (1997) Parametrization in laminate design for optimal compliance. Int J Solids Struct 34(4):415-434

[12] Hyer MW, Lee HH (1991) The use of curvilinear fiber format to improve buckling resistance of composite plates with central circular holes. Compos Struct 18:239-261

[13] Reddy JN (2003) Mechanics of composite laminated plates and shells: theory and analysis. Boca Raton, FL, CRC Press

[14] Kim BC, Weaver PM, Potter K (2014) Manufacturing characteristics of the continuous tow shearing method for manufacturing of variable angle tow composites. Compos Part A-Appl S 61:141-151

[15] Kim BC, Weaver PM, Potter K (2015) Computer aided modelling of variable angle tow composites manufactured by continuous tow shearing. Compos Struct 129:256267

[16] Mathworks (2016) Optimization Toolbox. http://it.mathworks.com/help/optim/index.html

[17] Montemurro M (2012) Optimal design of advanced engineering modular systems through a new genetic approach. PhD thesis, UPMC, Paris VI, France. http://tel .archives-ouvertes.fr/tel-00955533

[18] Montemurro M, Vincenti A, Vannucci P (2012) Design of elastic properties of laminates with minimum number of plies. Mech Compos Mater 48:369-390 
[19] Montemurro M, Vincenti A, Vannucci P (2012) A two-level procedure for the global optimum design of composite modular structures - application to the design of an aircraft wing. Part 1: theoretical formulation. J Optimiz Theory App 155(1):1-23

[20] Montemurro M, Vincenti A, Vannucci P (2012) A two-level procedure for the global optimum design of composite modular structures - application to the design of an aircraft wing. Part 2: numerical aspects and examples. J Optimiz Theory App 155(1):2453

[21] Montemurro M, Vincenti A, Vannucci P (2013) The automatic dynamic penalisation method (ADP) for handling constraints with genetic algorithms. Comput Method Appl M 256:70-87

[22] Montemurro M (2015) An extension of the polar method to the first-order shear deformation theory of laminates. Compos Struct 127:328-339

[23] Montemurro M (2015) Corrigendum to" an extension of the polar method to the first- order shear deformation theory of laminates"[Compos Struct 127 (2015) 328339]. Compos Struct 131:1143-1144

[24] Montemurro M (2015) The polar analysis of the third-order shear deformation theory of laminates. Compos Struct 131:775-789

[25] Montemurro M, Catapano A, Doroszewski D (2016) A multi-scale approach for the simultaneous shape and material optimisation of sandwich panels with cellular core. Compos Part B-Eng 91:458-472

[26] Nagendra S, Kodiyalam S, Davis J, Parthasarathy V (1995) Optimization of tow fiber paths for composite design. In: Proceedings of the AIAA/ASME/ASCE/AHS/ASC 36th Structures, Structural Dynamics and Materials Conference, vol. AIAA 95-1275, New Orleans, LA

[27] Nagendra S, Jestin D, Gürdal Z, Haftka RT, Watson LT (1996) Improved genetic algorithm for the design of stiffened composite panels. Comput Struct 58(3):543-555

[28] Nik MA, Fayazbakhsh K, Pasini D, Lessard L (2012) Surrogate-based multi-objective optimization of a composite laminate with curvilinear fibers. Compos Struct 94:23062313

[29] Nik MA, Fayazbakhsh K, Pasini D, Lessard L (2014) Optimization of variable stiffness composites with embedded defects induced by automated fiber placement. Compos Struct 107:160-166 
[30] Parnas L, Oral S, Ceyhan U (2003) Optimum design of composite structures with curved fiber courses. Compos Sci Technol 63:1071-1082

[31] Peeters DMJ, Hesse S, Abdalla MM (2015) Stacking sequence optimisation of variable stiffness laminates with manufacturing constraints. Compos Struct 125:596-604

[32] Piegl L, Tiller W (1997) The NURBS Book. Springer-Verlag

[33] Raju G, Wu Z, Kim BC, Weaver PM (2012) Pre-buckling and buckling analysis of variable angle tow plates with general boundary conditions. Compos Struct 94:29612970

[34] Setoodeh S, Abdalla MM, Gürdal Z (2006) Design of variable stiffness laminates using lamination parameters. Compos Part B-Eng 37:301-309

[35] Setoodeh S, Abdalla MM, IJsselmuiden ST, Gürdal Z (2006) Design of variable stiffness composite panels for maximum buckling load. Compos Struct 87:109-117

[36] Van Campen JMJF, Kassapoglou C, Gürdal Z (2011) Generating realistic laminate fiber angle distributions for optimal variable stiffness laminates. Compos Part B-Eng $43(2): 354-360$

[37] Vannucci P (2005) Plane anisotropy by the polar method. Meccanica 40:437-454

[38] Vannucci P (2013) A note on the elastic and geometric bounds for composite laminates. J Elasticity 112:199-215

[39] Verchery G (1979) Les invariants des tenseurs d'ordre 4 du type de l'élasticité. In: Proc. of Colloque Euromech 115, VIllard-de-Lans, France

[40] Wu Z, Raju G, Weaver PM (2015) Framework for the buckling optimisation of variable-angle-tow composite plates. AIAA J 53(12):3788-3804 